\title{
Delayed Nipple-Sparing Modified Subcutaneous Mastectomy: Rationale and Technique
}

\author{
Beniamino Palmieri, MD, ${ }^{*}$ George Baitchev, $\mathrm{MD},{ }^{\dagger}$ Simone Grappolini, $\mathrm{MD},{ }^{\ddagger}$ \\ Alberto Costa, MD, ${ }^{\mathbb{S}}$ and Giorgia Benuzzi, MD* \\ *Department of General Surgery, University of Modena, Modena, Italy; ${ }^{\dagger}$ Breast Unit, University Cancer \\ Center, Pleven, Bulgaria; ${ }^{\ddagger}$ Plastic Surgery Unit, Humanitas Institute, Rozzano, Italy; and ${ }^{\mathbb{B}}$ Breast Unit, \\ Fondazione Maugeri Hospital, Pavia, Italy
}

Abstract: The problem of nipple-areola complex (NAC) preservation during mastectomy is a very intriguing and stimulating issue. In fact, in order to perform an oncologically safe operation, no mammary tissue (enclosed in the main galactophoric ducts) should remain; on the other hand, without the blood supply coming from the breast gland, NAC viability is greatly impaired because the surrounding vascular dermal network is not developed enough to support its metabolic requirements. We suggest therefore a two-step surgical procedure. The first step, on an outpatient basis with local tumescent anesthesia, is a mini-invasive cutting and coagulating procedure. It addresses the autonomization of the vascular supply to the NAC by detaching the galactophore stalk from the nipple and coagulating the deep vascular plexus. The second step, under general anesthesia and again with tumescent technique, removes the breast within its capsule, with careful checks of any remnant and adequate approach to the axilla. A subpectoralis prosthesis completes the procedure. In our view, this technique is electively suitable for prophylactic mastectomy, but also for stage I breast cancer, $2.5 \mathrm{~cm}$ from the NAC and $1.5 \mathrm{~cm}$ from the skin and pectoralis fascia, and it is very safe, simple, and effective. .

Key Words: delayed nipple-sparing modified subcutaneous mastectomy, mastectomy, nipple-areola complex preservation

$\mathrm{T}$ he inheritable breast cancer recently detectable by evidence of the BRCA-1/BRCA-2 gene mutation, lobular hyperplasia, atypical ductal hyperplasia, and lobular carcinoma in situ are currently faced on a preventive basis with selective screening and follow-up, chemoprevention, and/or prophylactic mastectomy (1-4). The surgical options of simple mastectomy and subcutaneous mastectomythe former excising the nipple-areola complex (NAC) together with the gland, the latter leaving intact the NACprovides $95-99 \%$ and $90-95 \%$ breast tissue removal, respectively, and thus being inadequate as to oncologic radicality (5). Therefore when risk of breast cancer is high, total mastectomy is the golden standard.

A $13 \%$ increase (from $81 \%$ to $94 \%$ ) of total mastectomies in cohorts of women with a family history of breast malignancy has been observed since 1995 , followed by an high rate of reconstruction. Metcalfe et al. (6) report breast restoration in $60 \%$ of these patients, compared with $6-13 \%$ of nonprophylactic mastectomies.

Address correspondence and reprint requests to: Beniamino Palmieri, MD, University of Modena and Reggio Emilia, Department of General Surgery and Surgical Specialty, Surgical Clinics, Via del Pozzo 71, 41100 Modena, Italy, or email: palmieri@unimo.it.

(๐) 2005 Blackwell Publishing, Inc., 1075-122X/05

The Breast Journal, Volume 11 Number 3, 2005 173-178
Our study addresses the hypothesis of a new radical approach to subcutaneous mastectomy while retaining the integrity of the NAC, without leaving any gland stalk or parenchyma underneath, and thus obtaining complete clearance of the breast tissue. Primary reconstruction with subpectoralis prosthesis or two-stage replacement with an expander are the surgical options to complete the procedure in order to achieve satisfactory cosmetic results. This procedure addresses both cancer prophylaxis and stage I cancer treatment.

\section{MATERIALS AND METHODS}

From April 1999 to August 2004, we operated on 18 women (Table 1), age 29-62 years, recruited on the basis of the following guidelines: T1 cancer, $2.5 \mathrm{~cm}$ from the $\mathrm{NAC}$ and at least $1.5 \mathrm{~cm}$ from the skin and pectoralis fascia. All patients signed an informed consent form with analytical details of the procedure. The procedure was divided into two different phases: NAC vascular autonomization on an outpatient basis using local tumescent anesthesia with laparoscopic instrumentation; and delayed nipple-sparing modified subcutaneous mastectomy using general anesthesia with tumescent technique plus subpectoralis textured silicone breast implant. 
Table 1. Patient Information

\begin{tabular}{|c|c|c|}
\hline Patient no. & Age & Description \\
\hline 1 & 34 & Left contralateral lobular cancer. The patient had a previous right quadrantectomy and radiotherapy) (pT1N0M0) \\
\hline 2 & 42 & Left breast cancer and fibrocystic mastopathy with axillary metastasis who refused total mastectomy (bilateral) ( $\mathrm{pT} 1 \mathrm{~N} 1 \mathrm{M} 0)$ \\
\hline 3 & 37 & Fibrocystic mastopathy and familiar breast cancer (bilateral) \\
\hline 4 & 39 & Bilateral previous quadrantectomy plus previous radiation therapy to one side (bilateral) \\
\hline 5 & 62 & Breast dysplasia and previous contralateral mastectomy \\
\hline 6 & 42 & Severe bilateral relapsing mastitis (bilateral) \\
\hline 7 & 51 & Lobular carcinoma in situ (left breast) and ductal carcinoma in situ (right) (bilateral) \\
\hline 8 & 56 & Right lower lateral quadrant ductal infiltrating carcinoma (pT1N0M0) \\
\hline 9 & 44 & Painful fibrocystic disease and breast cancer in mother and older sister (bilateral) \\
\hline 10 & 37 & Right infiltrating ductal carcinoma (pT1N1M0) \\
\hline 11 & 42 & Right breast infiltrating ductal carcinoma (pT1N1M0) \\
\hline 12 & 61 & Right breast comedo carcinoma (pT1N1M0) \\
\hline 13 & 58 & Right breast infiltrating ductal carcinoma (pT1NOM0) \\
\hline 14 & 29 & Right breast lobular carcinoma (pT1N1M0) \\
\hline 15 & 48 & Right breast infiltrating ductal carcinoma (pT1NOM0) \\
\hline 16 & 30 & Cystic mastopathy and family cancer (bilateral) \\
\hline 17 & 35 & Left breast solid carcinoma (pT1N1M0) \\
\hline 18 & 45 & Right breast infiltrating ductal carcinoma (pT1N0M0) \\
\hline
\end{tabular}

\section{Details of the Procedure}

Phase 1 consists of premedication with diazepam 10 $\mathrm{mg}$ intramuscularly, tramadol $100 \mathrm{mg}$, and cefazolin $1 \mathrm{~g}$ (Fig. 1A). In the supine position, the areola-periareolar subcutaneous tissue (each side) is infiltrated using a 21gauge needle with anesthetic solution: 1\% Carbocaine and epinephrine (1:1000) in $20 \mathrm{ml}$ and $80 \mathrm{ml}$ saline with $10 \mathrm{ml}$ sodium bicarbonate (1:16) (Fig. 1B).

The orange-peel skin is obtained over a $20 \mathrm{~cm}$ diameter. After 20 minutes, when the skin is blanched due to vasoconstrictor effect, a $5 \mathrm{~mm}$ hole is placed $18 \mathrm{~cm}$ from the nipple with a no. 11 blade along the line of approach for the final operation. Electrified laparoscopic scissors are then introduced through this small incision and dissection of the periareolar skin and nipple-areola from the breast gland stalk is completed on a very surficial subcutaneous plane (Fig. 1C). The breast parenchyma is totally severed from the skin and coagulated, leaving only the dermal vascular network to supply the NAC.

Great care is taken to avoid skin overheating or burning: sponges filled with cold water are applied to the skin surface and cold saline irrigation mixed with $1 \mathrm{~g}$ chloramphenicol powder is introduced in the subcutaneous space (Fig. 1D).

Careful hemostasis control follows this step, with dry gauze strips introduced into the wound up to the subareolar dead space to detect and stop any blood leaking. Microscopic examination of the major ductal breast stalk is now possible to evaluate potential tumor seeding.

Finally, six to eight fine strips of collagen $(5 \mathrm{~cm} \times 0.5$ $\mathrm{cm})$ with gentamicin are inserted to insulate the recently dissected deep subcutaneous surface from the vascular network of the cut gland (Fig. 1E). A single Ethilon stitch is used to close the skin.

The breasts are wrapped in an elastic bandage. The patient is given analgesic and antibiotic prescriptions for 3 days. Stitch removal is performed 6 days later.

Phase 2 occurs 3 weeks later, when the patient is admitted to the hospital for the final mastectomy or mastectomy plus lymphadenectomy. Under general anesthesia, the breast subcutaneous fat close to the dermis (bilaterally) is infiltrated with saline solution $(250 \mathrm{ml}$ each) with antibiotic and $0.25 \mathrm{mg}$ epinephrine, producing a widespread orange-peel skin appearance.

When the surface starts to be pale due to vasoconstriction, a $12-15 \mathrm{~cm}$ incision is made, either vertically between the internal and external quadrants or laterally between the upper and lower quadrants (Fig. 2A). In cases where a previous scar exists, the incision overlaps it to minimize vascular injury to the NAC.

The surgeon reaches the breast capsule and starts detaching it from the subcutaneous stroma with blunt dissection and coagulation, paying attention not to overheat the dermal vessels and to avoid tearing the capsule (Fig. 2B). Special care is taken with the axillary prolongation excision; in the case of axillary and/or sentinel lymph node dissection, the lateral incision is preferred because of the optimal vessels and nerve exposure.

When the dead subareolar space is approached, the surgeon checks the inner surface of the nipple-areola as well as the coagulated breast gland stalk to clear loose collagenic adhesions; at this time subcutaneous biopsy of the nipple remnant is performed and samples of 

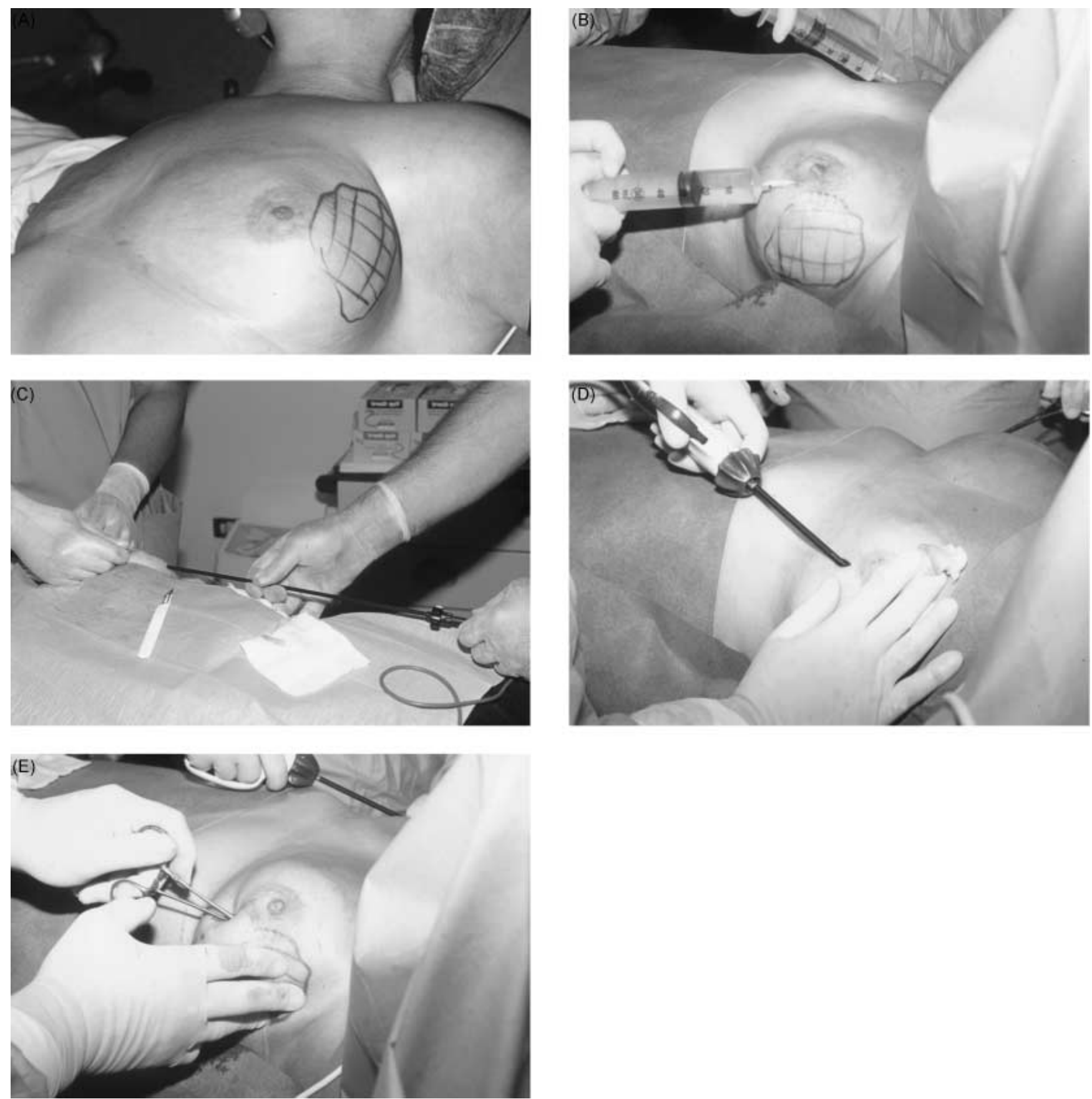

Figure 1. Phase 1. (A) Cystic mastopathy and ductal infiltrating carcinoma, preoperative view. (B) Local anesthesia and tumescent injection to induce orange-peel skin. (C) Electrified laparoscopic equipment. (D) Vascular network coagulation and galactophore stalk dissection: the NAC skin is cooled by wet sponges. (E) Blind final adjustment of the operative field that is covered with several collagen fleeces in order to prevent regeneration of the cut and coagulated vessels; the collagen is inserted with a small vascular clamp.

the galactophoric stalk are prepared and sent to the pathologist.

Finally, after having removed the subcutaneous surface of the breast capsule, it is possible to draw out the entire gland (Fig. 2C). The pectoralis fascia is preserved or excised, with accurate control of the bleeding (Fig. 2D).
At this point, a very accurate bench examination of the breast is done (Fig. 2E), with special attention to the capsule integrity, then a second look with breast skin fiberoptic transillumination or a laparoscopic camera is performed to be sure that no remnants or succenturiate lobules have been inadvertently left in place. In case of 

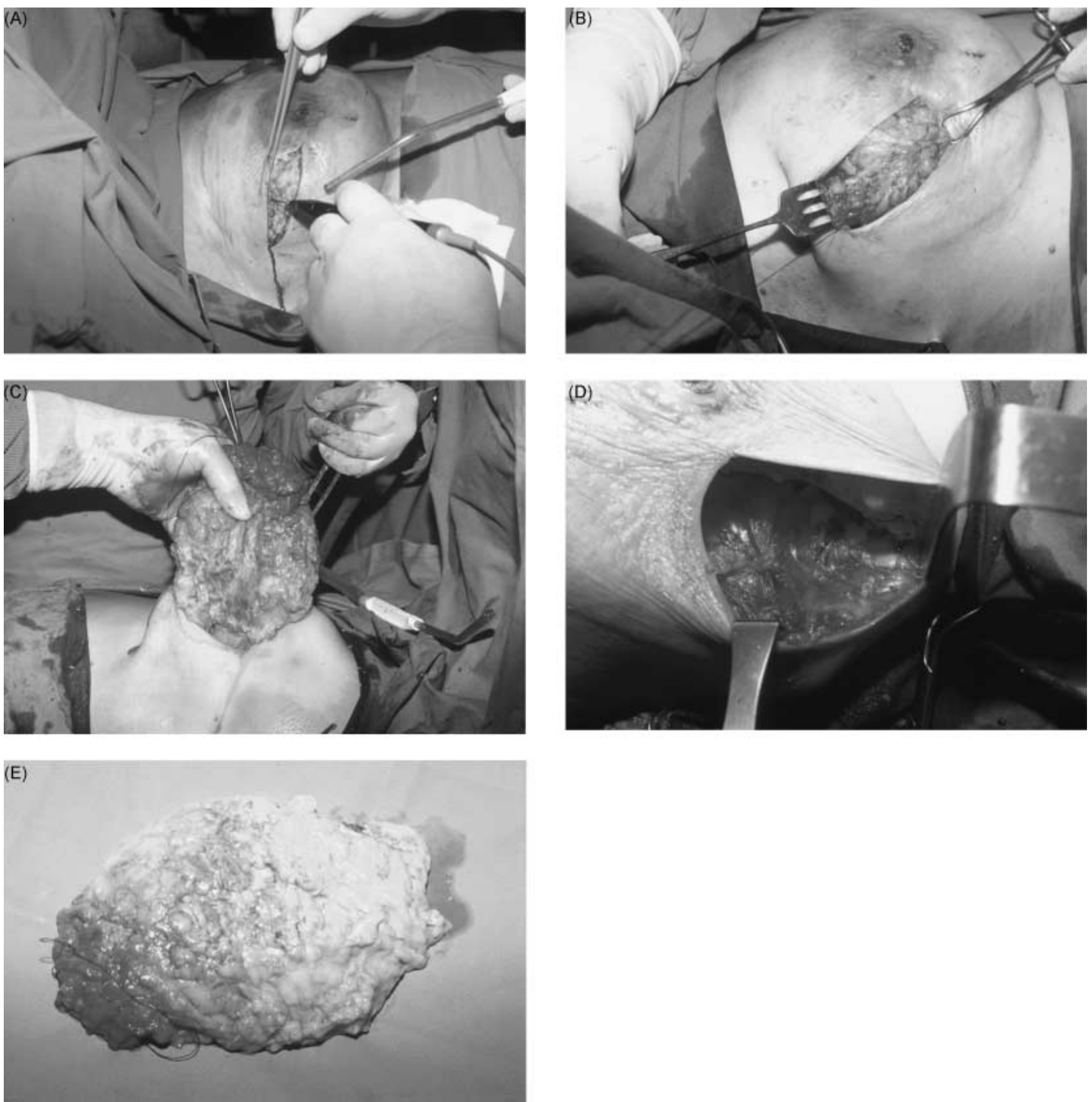

Figure 2. Phase 2. (A) Lateral incision to achieve the best tumor and axillary exposure: note the NAC viability. (B) Mobilization of the gland capsule from the dermis thickened by the tumescent technique. (C) The whole breast is delivered from the incision at the end of the deep dissection. (D) A clean thoracic surface after radical nipple-sparing mastectomy: under the nipple-areola there is only regular dermal tissue whose oncologic stage can be verified by slide imprint cytology or biopsy. (E) Gross specimen appearance: there is no damage to the breast capsule.

tumor surgery, 12 surgical biopsies ( 3 for each quadrant) of the subcutaneous fat tissue are sent to the pathologist to confirm the absence of any gland remnant or tumor cluster. When clearance is confirmed and hemostasis is achieved, a subpectoralis silicone prosthesis or temporary skin expander can be placed to prepare the pocket for reconstruction. Suction drains are inserted and left in place for 72 hours.

Pectoralis muscle has to be completely detached from lateral and distal insertion, and a properly sized textured 
(A)

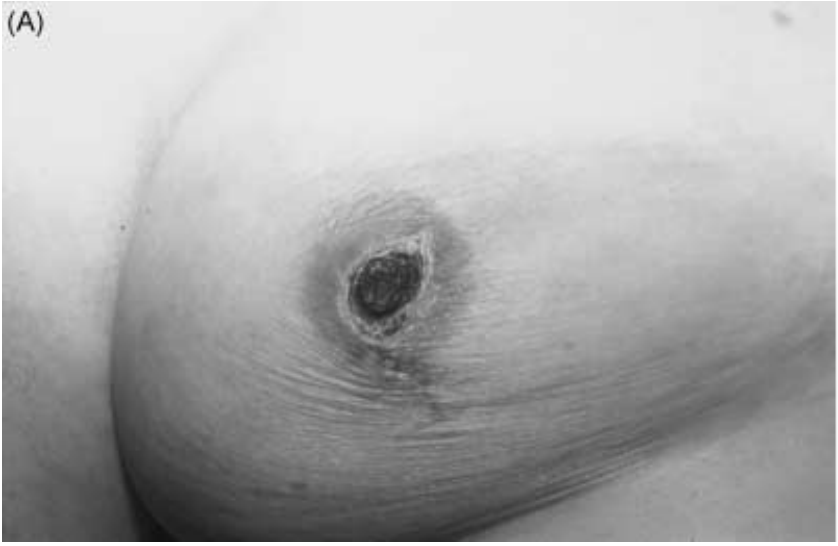

(B)

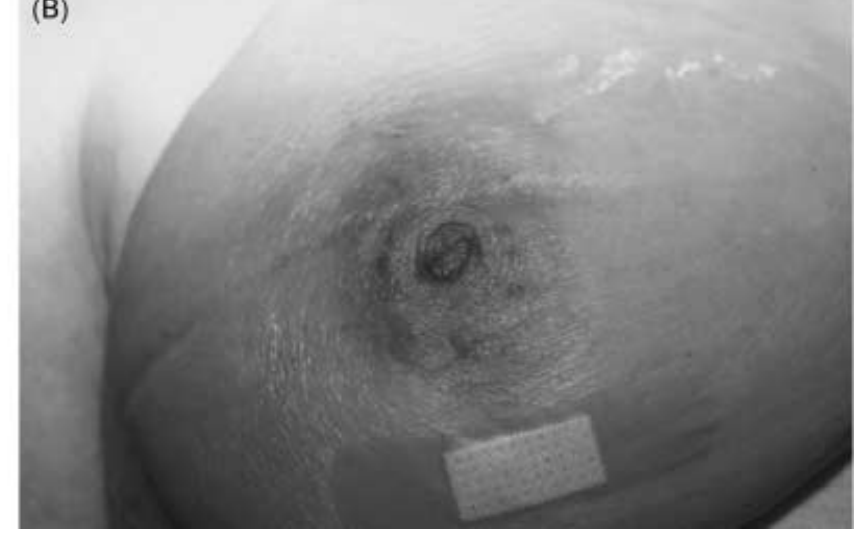

Figure 3. (A) Incorrect and (B) correct outcome of NAC autonomization performed by two surgeons on the same patient at the same time: overheating and burning of the nipple stalk produces coagulative necrosis that heals in 4-8 weeks.

silicone prosthesis (Eurosilicone, France) is wrapped within its fibers with absorbable stitches to avoid any direct contact between the skeletonized skin and the polysiloxane membrane. Proper skin closure and elastic bandaging complete the procedure.

\section{RESULTS}

No NAC necrosis has been observed, except in patient 1 , during the learning curve training, where overheating caused heat injury to the skin (Fig. 3). In this patient, the second operation was postponed 6 weeks to allow good scar formation and dermal vascular network regeneration. The final outcome was satisfactory. No depigmentation or other damage to the NAC has ever been observed.

We have also been able to safely lift the NAC during the reconstruction phase in two cases, allowing us to achieve symmetry.

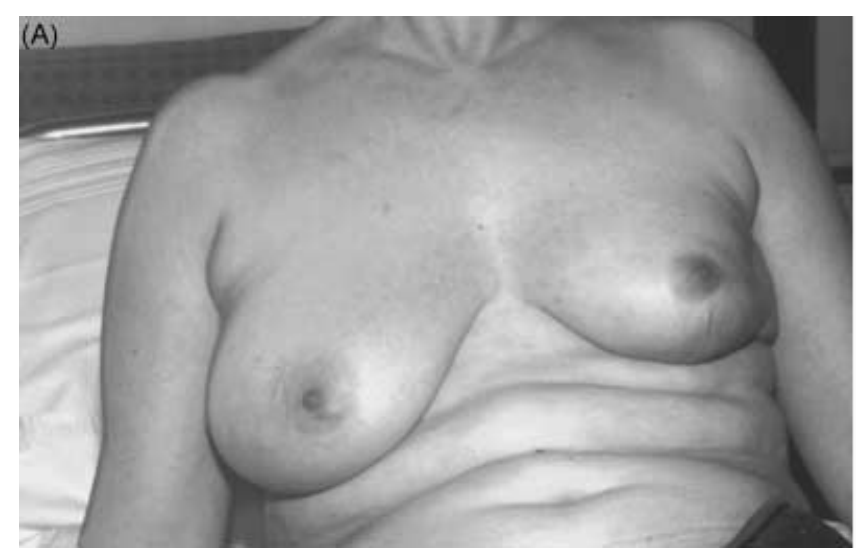

In one case (patient 2) we didn't immediately insert the prosthesis because of planned chemoradiotherapy. The NAC was undamaged and the patient is awaiting reconstruction.

Another case had unilateral delayed nipple-sparing modified subcutaneous mastectomy and replacement with prosthesis, but not contralateral mastopexy (Fig. 4).

No local or axillary tumor relapse has been observed, with an average follow-up of 21 months (range 6-52 months).

\section{DISCUSSION}

Our results show that the two-step delayed nipplesparing modified subcutaneous mastectomy is a safe, simple, and effective procedure to completely remove the gland with intraoperative direct determination of any possible remnant. The autonomization of the NAC obtained

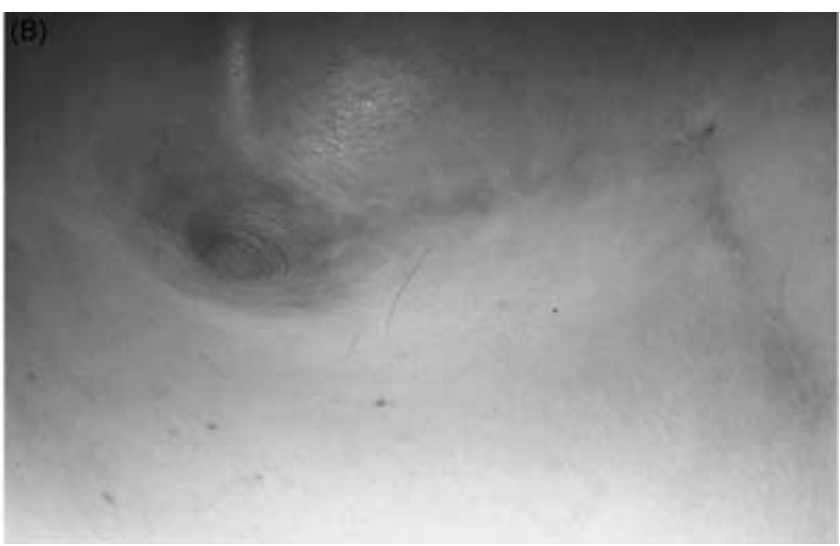

Figure 4. (A) Breast carcinoma detected 2 years after quadrantectomy far from the nipple: the scar of our operation overlaps the previous incision. (B) The same patient 3 months later: contralateral symmetric adjustment has been planned, but not yet done. 
with blind dissection by means of laparoscopic instruments leaves only the dermal plexus as the main vascular supply (7). This very fine network is able to guarantee the integrity of breast skin appendages (assuming that they are being cut very close to the dermis) and even to mobilize the nipple-areola if mastopexy is required during reconstruction.

Hemostasis of detached galactophores, antibiotic irrigation of the surgical field, and collagen fleece insulation of the deep nipple-areola layer are three main steps to prevent infections and complications and to induce an efficient collateral circulation and autonomization of the overlying skin: vascular reconnection from the breast gland to the skin is prevented and very loose adhesions are detected during the second part of the procedure. The opportunity to do cytologic smears or frozen sections of both the cut surfaces during the second surgical step gives further oncologic awareness of radicality, and is why this technique is also used for stage I breast cancer.

We believe this procedure is another option to classic skin-preserving mastectomies. This approach should be considered especially for young women with a high family cancer risk, because it takes equally into account complete gland removal and cosmetic reconstruction in a very simple way.

A two-step surgical approach, the first one being an office procedure, makes nipple-areola preservation easy and safe. Furthermore, with this technique, accurate pathologic details as to the absence of cancer cells under the NAC are obtained at two different times. The safe interval between the first and second operation is $2-4$ weeks, based on the time required to open the collateral capillary network and complete the newly formed angiogenetic processes.

This simple method achieves vascular autonomization and oncologic safety of the preserved NAC. Wider patient recruitment and long-term follow-up of this technique are needed.

\section{Acknowledgments}

We thank Nathan Smith and Kyle Jardine for reviewing the manuscript.

\section{REFERENCES}

1. Klijn JG, Janin N, Cortes-Funes H, Colomer R. Should prophylactic surgery be used in women with a high risk of breast cancer? Eur J Cancer 1997;33:2149-59.

2. Hartmann LC, Schaid DJ, Woods JE, et al. Efficacy of bilateral prophylactic mastectomy in women with a family history of breast cancer. N Engl J Med 1999;340:77-84.

3. Josephson U, Wickman M, Sandelin K. Initial experiences of women from hereditary breast cancer families after bilateral prophylactic mastectomy: a retrospective study. Eur J Surg Oncol 2000;26:351-56.

4. Meijers-Heijboer H, van Geel B, van Putten WL, et al. Breast cancer after prophylactic bilateral mastectomy in women with a BRCA1 or BRCA2 mutation. N Engl J Med 2001;345:159-64.

5. Crane R. Breast cancer. In: Otto SE, ed. Oncology Nursing, 2nd ed. St. Louis: Mosby, 1994:90-129.

6. Metcalfe KA, Goel V, Lickley L, Semple J, Narod SA. Prophylactic bilateral mastectomy. patterns of practice. Cancer 2002;95:236-42.

7. Nakajima H, Imanishi N, Aiso S. Arterial anatomy of the nippleareola complex. Plast Reconstr Surg 1995;96:843-45.

8. Worland RG. Expanded utilization of the tumescent technique for mastectomy. Plast Reconstr Surg 1996;98:1321. 